\title{
Local Convergence of the Sequential Quadratic Method for Differential Games
}

\author{
Akio Tanikawa ${ }^{\dagger}$, Hiro Mukai ${ }^{\ddagger}$ and Min $\mathrm{Xu}^{\ddagger}$ \\ †Faculty of Information Science and Technology, \\ Osaka Institute of Technology \\ Kitayama, Hirakata-shi, 573-0196, Japan \\ E-mail: tanikawa@is.oit.ac.jp \\ $\ddagger$ Department of Electrical and Systems Engineering \\ Washington University \\ St. Louis, MO 63130 USA
}

\begin{abstract}
For computing a Nash (saddle point) solution to a zero-sum differential game for a general nonlinear system, Mukai et al. presented an iterative Sequential Quadratic-Quadratic Method (SQQM) as follows. Given a solution estimate, they defined a subproblem which approximates the original problem up to the second order around the solution estimate. They proposed to replace the subproblem with another subproblem in order to obtain a game problem with only a linear dynamics by removing the quadratic terms in the system dynamics and adding them to the payoff function as in Lagrangian function. We can now solve this subproblem conveniently by a Riccati equation method. We then update the solution estimate by adding its Nash solution to the current solution estimate for the original game. Through our extensive experiments, we observed not only local convergence of the SQQM but also much faster convergence of the SQQM than the iterative methods based on lower order approximations such as the Sequential Linear-Quadratic Method (SLQM). In this paper we will establish local convergence of the SQQM.
\end{abstract}

Keywords: differential games, saddle points, Riccati equations.

\section{Introduction}

In paper [8], our group presented an iterative procedure, the Sequential Quadratic-Quadratic Method (SQQM), for computing a local Nash (saddle point) equilibrium solution to a general zero-sum differential game, in which the system dynamics is not necessarily linear and the payoff function is not necessarily quadratic. We briefly review the basic idea of the SQQM in the following. Given a solution estimate to the general zero-sum differential game, it is quite nat-

\footnotetext{
${ }^{1}$ Part of this research was supported by the Defense Advanced Research Projects Agency (DARPA) and Air Force Research Laboratory, Air Force Materiel Command, USAF, under agreement number F30602-99-2-0551 and also by the Japan Society for Promotion of Science (JSPS) under Grant-in Aid for Scientific Research (C)-22540158.
}

ural to define a subproblem which approximates the original game problem by applying quadratic expansions to both the system dynamics and the payoff function around the current solution estimate. Solving this subproblem and adding its solution to the current solution estimate, we may expect to have a new improved solution estimate to the original game problem. However, each approximated subproblem cannot be solved easily since the subproblem does not have a linear (or affine) dynamics. Thus, in order to avoid this difficulty, the linear (or affine) approximation to the system dynamics was actually proposed in the Sequential LinearQuadratic Method (SLQM) in [6, 7]. Later in [8], this drawback was overcome as follows: we proposed a new subproblem by removing the quadratic terms in the system dynamics and adding them to the payoff function after multiplied by a suitable multiplier function. This idea was inspired by the pioneering work of Wilson [13] and also the works of Robinson [9, 10]. Because the new subproblem has only a linear dynamics, we can now apply a Riccati equation method and compute the Nash solution to the subproblem. We then add this Nash solution to the current solution estimate for the original game. This sum becomes a new solution estimate for the original game. Repeating this procedure, we successively generate a sequence of solution estimates.

Our experiments [8] showed that the sequence of solution estimates generated by the SQQM converges to a local Nash solution of the original game. However, the proof for its convergence was missing. We also observed much faster convergence of the SQQM than the iterative methods based on lower order approximations such as the SLQM reported in $[6,7]$. This faster convergence in numerical experiments seems to verify that the subproblem of SQQM, although implemented as a linearly-constrained quadratic problem, retains the intrinsic characteristic of a quadratic approximation around the solution estimate to the original game problem.

The purpose of this paper is to show local convergence of the SQQM. In fact, we prove that the iterative process SQQM is asymptotically equivalent to the Newton's method for solving an equation $F(z)=0$, for some nonlinear operator $F$ on a normed linear space into a Banach space. We shall prove linear conver- 
gence in Theorem 4.2 and quadratic convergence under stronger conditions in Theorem 4.3.

In Section II, we formulate our problem as a general differential game, i.e., both the system dynamics and the payoff function are not necessarily quadratic, and we review the iterative method, SQQM, for finding the Nash equilibrium solution to the nonlinear differential game. After presenting some preliminary results in Section III, we prove the main results in Section IV.

\section{PROBLEM FORMULATION AND THE SQQM}

Our research started from the analysis of an aerial war game between two air force squadrons. Since the outcome of any combat is stochastic in nature. We derived the following vector differential equation driven by random vectors which describe our combat game.

Let $U$ denote the space of continuous functions from $\left[t_{0}, t_{f}\right]$ into $\mathbf{R}^{m}$. Let us consider the following stochastic system:

$$
\frac{d}{d t} x(t)=f(x(t), u(t))+w(t), t \in\left[t_{0}, t_{f}\right] ; x\left(t_{0}\right)=x_{0},
$$

where $f(x, u)$ is a $C^{2}$-class function from $\mathbf{R}^{n} \times \mathbf{R}^{m}$ into $\mathbf{R}^{n}$ and $w(t)$ is a stationary white Gaussian process. In [3] we considered the differential game for (1):

$$
J^{*}=\min _{u^{B}} \max _{u^{R}}\left\{J(u) \mid \frac{d}{d t} x(t)=f(x(t), u(t))+w(t)\right\},
$$

where the control function $u$ consists of two parts, $u^{B}$ and $u^{R}$, corresponding to the two forces, the Blue and the Red: $u=\left(u^{B}, u^{R}\right)$ and the Red force tries to maximize the payoff function $J\left(u^{B}, u^{R}\right)$ locally and the Blue force tries to minimize the same payoff function $J\left(u^{B}, u^{R}\right)$ locally. The payoff function $J\left(u^{B}, u^{R}\right)$ will be explicitly given later.

We quickly realized that the timely numerical solution of the above stochastic game will not be possible for the air combat applications in which solutions are needed within seconds. We compared [11] the expected states from continuous-transition Markov chain probabilistic models of air combats between two opposing squadrons and the states of the deterministic models based on the ordinary differential equations without random vectors which approximate the dynamics of the expected states of the stochastic models. We found [11] that the deterministic model approximated the stochastic model well enough.

We furthermore proposed a state estimator for system (1) which can be constructed without knowing enemy inputs in [3]. We found that the estimator works well and so the effect of the random disturbance $w(t)$ can be ignored without too much loss in accuracy in the process of obtaining the game solution of (2).
We thus consider the following dynamical system whose noise has been removed from (1):

$$
\frac{d}{d t} x(t)=f(x(t), u(t)), t \in\left[t_{0}, t_{f}\right] ; \quad x\left(t_{0}\right)=x_{0},
$$

where $f(x, u)$ is a $C^{2}$-class function from $\mathbf{R}^{n} \times \mathbf{R}^{m}$ into $\mathbf{R}^{n}$. Given any control $u \in U$ and an initial state $x\left(t_{0}\right)=x_{0}$, we assume that equation (3) defines a unique continuously differentiable solution $x(t), t \in$ $\left[t_{0}, t_{f}\right]$, which is called the trajectory of the system produced by control $u$ and denoted also by $x[u] \in C^{1}\left[t_{0}, t_{f}\right]$, where $C^{1}\left[t_{0}, t_{f}\right]$ is the space of continuously differentiable functions from $\left[t_{0}, t_{f}\right]$ into $\mathbf{R}^{n}$.

As a payoff function, we consider

$J(u)=J\left(u^{B}, u^{R}\right)=\int_{t_{0}}^{t_{f}} g(x(t), u(t)) d t+g_{f}\left(t_{f}, x\left(t_{f}\right)\right)$

where $g$ and $g_{f}$ can be general $C^{2}$-class functions. The overall game is expressed as the following minimax problem:

$$
J^{*}=\min _{u^{B}} \max _{u^{R}}\left\{J(u) \mid \frac{d}{d t} x=f(x, u), x\left(t_{0}\right)=x_{0}\right\} .
$$

The optimal value $J^{*}$ of the payoff function $J\left(u^{B}, u^{R}\right)$ is called the value of the game. Recall that the control function $u^{*}=\left(u^{* B}, u^{* R}\right)$ is a (local) Nash (saddle point) solution to game problem (4) if it satisfies the inequalities

$$
J\left(u^{* B}, v^{R}\right) \leq J\left(u^{* B}, u^{* R}\right) \leq J\left(v^{B}, u^{* R}\right),
$$

for any $v=\left(v^{B}, v^{R}\right)$ in a neighborhood of $u^{*}$. The optimal value $J^{*}\left(t_{0}, x_{0}\right)=J\left(u^{*}\right)$ of the objective function $J\left(u^{B}, u^{R}\right)$ is called the value of the game and it depends on the initial time $t_{0}$ and the initial state $x_{0}$.

For a given adjoint function $\lambda \in C^{1}\left[t_{0}, t_{f}\right]$, we define the Hamiltonian $H$ of game (4) by

$$
H(x, u, \lambda)=g(x, u)+\lambda^{\prime} f(x, u),
$$

where we denote transposition by a prime.

In order to simplify the minimax problems in game (4) and the subproblems which approximate game (4), we make the following assumption [8].

\section{Assumption 1}

$$
\begin{gathered}
g_{u^{B} u^{R}}\left(x, u^{B}, u^{R}\right) \equiv 0, g_{u^{R} u^{B}}\left(x, u^{B}, u^{R}\right) \equiv 0, \\
f_{u^{B} u^{R}}\left(x, u^{B}, u^{R}\right) \equiv 0, f_{u^{R} u^{B}}\left(x, u^{B}, u^{R}\right) \equiv 0,
\end{gathered}
$$

where $g_{u^{B} u^{R}}$ denote the partial derivative of $g$ with respect to $u^{B}$ and $u^{R}$, and so on.

Roughly speaking, assumption (5) states that there are no cross product terms between the Blue and Red controls in the payoff function. Similarly, assumption (6) states that there are no cross product terms between Blue and Red controls in the righthand side of 
the differential equations. With these assumptions, the following conditions hold over $\left[t_{0}, t_{f}\right]$ :

$H_{u^{B} u^{R}}\left(x^{*}, u^{* B}, u^{* R}, \lambda^{*}\right) \equiv H_{u^{R} u^{B}}\left(x^{*}, u^{* B}, u^{* R}, \lambda^{*}\right) \equiv 0$.

Suppose that there exists a local Nash solution $u^{*}(t)=\left(u^{* B}(t), u^{* R}(t)\right)$ to game (4). Then, there exist a unique state trajectory $x^{*}(t)=x\left[u^{*}\right](t)$ and a unique adjoint function $\lambda^{*}(t)$ (see Section 9.3 of [2] or Theorem 6.13 of [1]) such that for $t \in\left[t_{0}, t_{f}\right]$

$$
\begin{aligned}
& \frac{d}{d t} x^{*}(t)=f\left(x^{*}(t), u^{*}(t)\right), \quad x^{*}\left(t_{0}\right)=x_{0}, \\
& \frac{d}{d t} \lambda^{*}(t)=-H_{x}\left(x^{*}(t), u^{*}(t), \lambda^{*}(t)\right)^{\prime}, \\
& \lambda^{*}\left(t_{f}\right)=\left(g_{f}\right)_{x}\left(x^{*}\left(t_{f}\right)\right)^{\prime}, \\
& H_{u}\left(x^{*}(t), u^{*}(t), \lambda^{*}(t)\right)=0,
\end{aligned}
$$

and moreover the following positive and negative semidefiniteness holds for each time $t \in\left[t_{0}, t_{f}\right]$,

$H_{u^{B} u^{B}}\left(x^{*}, u^{* B}, u^{* R}, \lambda^{*}\right) \geq 0, H_{u^{R} u^{R}}\left(x^{*}, u^{* B}, u^{* R}, \lambda^{*}\right) \leq 0$,

which corresponds to the Legendre-Clebsch condition (see Section 9.3 of [2]). For uniqueness of (local) Nash solution, we make use of the following slightly stronger positive and negative definiteness assumption:

Assumption 2 At each time $t \in\left[t_{0}, t_{f}\right]$,

$H_{u^{B} u^{B}}\left(x^{*}, u^{* B}, u^{* R}, \lambda^{*}\right)>0, H_{u^{R} u^{R}}\left(x^{*}, u^{* B}, u^{* R}, \lambda^{*}\right)<0$.

We use the notation $\lambda[u](t)$ for the solution $\lambda(t)$ of the ordinary differential equation, where $x=x[u]$ :

$\frac{d}{d t} \lambda(t)=-H_{x}(x(t), u(t), \lambda(t))^{\prime}, \lambda\left(t_{f}\right)=\left(g_{f}\right)_{x}\left(x\left(t_{f}\right)\right)^{\prime}$.

It immediately follows from (7), (8) and (9) that $x^{*}=$ $x\left[u^{*}\right]$ and $\lambda^{*}=\lambda\left[u^{*}\right]$. We also note that the matrix $H_{u u}(x(t), u(t), \lambda(t))$ is invertible in a neighborhood of $\left(x^{*}(t), u^{*}(t), \lambda^{*}(t)\right)$.

We now review the Sequential Quadratic-Quadratic Method (SQQM) given in [8]. We assume that a solution estimate triplet $\left(x_{i}, u_{i}, \lambda_{i}\right)$ with $x_{i}=x\left[u_{i}\right]$ and $\lambda_{i}=$ $\lambda\left[u_{i}\right]$ is available, and consider the following subproblem which can be obtained by applying quadratic approximation around the solution estimate $\chi_{i}=\left(x_{i}, u_{i}\right)$ to the original game problem, where the quadratic terms in the system dynamics multiplied by $\lambda_{i}$ was moved to the payoff function as in the Lagrangian function in order that the subproblem has only linear dynamics:

$$
\begin{aligned}
& \min _{\delta u^{B}} \max _{\delta u^{R}}\left\{\int _ { t _ { 0 } } ^ { t _ { f } } \left[g\left(\chi_{i}\right)+g_{x}\left(\chi_{i}\right) \delta x+g_{u}\left(\chi_{i}\right) \delta u\right.\right. \\
& +\frac{1}{2} \delta x^{\prime} g_{x x}\left(\chi_{i}\right) \delta x+\frac{1}{2} \delta u^{\prime} g_{u x}\left(\chi_{i}\right) \delta x+\frac{1}{2} \delta x^{\prime} g_{x u}\left(\chi_{i}\right) \delta u \\
& \left.+\frac{1}{2} \delta u^{\prime} g_{u u}\left(\chi_{i}\right) \delta u+\frac{1}{2} \sum_{j=1}^{n} \lambda_{i}^{(j)} \widetilde{f^{(j)}}\left(\chi_{i}\right)[\delta x, \delta u]\right] d t+g_{f}\left(x_{i}\left(t_{f}\right)\right) \\
& +\left(g_{f}\right)_{x}\left(x_{i}\left(t_{f}\right)\right) \delta x\left(t_{f}\right)+\frac{1}{2} \delta x\left(t_{f}\right)^{\prime}\left(g_{f}\right)_{x x}\left(x_{i}\left(t_{f}\right)\right) \delta x\left(t_{f}\right) \\
& \left.\frac{d}{d t} \delta x=f_{x}\left(\chi_{i}\right) \delta x+f_{u}\left(\chi_{i}\right) \delta u, \quad \delta x\left(t_{0}\right)=0\right\},
\end{aligned}
$$

where the second-order terms of the $j$-th component $f^{(j)}$ of the right-hand side are collected as

$\widetilde{f^{(j)}}[\delta x, \delta u]:=\delta x^{\prime} f^{(j)}{ }_{x x} \delta x+\delta u^{\prime} f^{(j)}{ }_{u x} \delta x+\delta x^{\prime} f^{(j)}{ }_{x u} \delta u+\delta u^{\prime} f^{(j)}{ }_{u u} \delta u$.

Here, the minimization (with respect to $\delta u^{B}$ ) and the maximization (with respect to $\delta u^{R}$ ) are respectively done in some neighborhoods of zero vectors (vectorvalued functions). Assumptions 1 and 2 are not enough to ensure the existence of local solution of subproblem (11). As sufficient conditions for existence of linearquadratic (or affine-quadratic) problems, we usually assume existence of solutions to some Riccati equations (see Chapter 6 of [1]). In this paper, we do not go into its details, but simply make the following assumption.

Assumption 3 Subproblem (11) has a unique solution $\delta u_{i}=\left(\delta u_{i}^{B}, \delta u_{i}^{R}\right)$.

The next lemma immediately follows from Assumption 3 (see, e.g., Theorem 6.13 of [1]).

Lemma 2.1 There exist a unique optimal solution $\delta u_{i}$ and the accompanying functions, $\delta x_{i}$ and $\gamma_{i+1}$, such that

$\frac{d}{d t} \delta x_{i}=f_{x}\left(\chi_{i}\right) \delta x_{i}+f_{u}\left(\chi_{i}\right) \delta u_{i}, \quad \delta x_{i}\left(t_{0}\right)=0$,

$\frac{d}{d t} \gamma_{i+1}=-H_{x}\left(\chi_{i}, \gamma_{i+1}\right)^{\prime}-H_{x x}\left(\chi_{i}, \lambda_{i}\right) \delta x_{i}-H_{x u}\left(\chi_{i}, \lambda_{i}\right) \delta u_{i},(13)$

$\gamma_{i+1}\left(t_{f}\right)=\left(g_{f}\right)_{x}\left(x_{i}\left(t_{f}\right)\right)^{\prime}+\left(g_{f}\right)_{x x}\left(x_{i}\left(t_{f}\right)\right) \delta x_{i}\left(t_{f}\right)$,

$H_{u}\left(\chi_{i}, \gamma_{i+1}\right)^{\prime}+H_{u u}\left(\chi_{i}, \lambda_{i}\right) \delta u_{i}+H_{u x}\left(\chi_{i}, \lambda_{i}\right) \delta x_{i}=0$.

Remark 2.2 (Consistency) If $\left(x_{i}, u_{i}\right)=\left(x^{*}, u^{*}\right)$, then

$$
\left(\delta x_{i}, \delta u_{i}, \gamma_{i+1}\right)=\left(0,0, \lambda^{*}\right) .
$$

Making use of the notation $\delta \lambda_{i}:=\gamma_{i+1}-\lambda_{i}$, we can rewrite equations (13) and (14) as follows.

Lemma 2.3 The ordinary differential equation (13)(14) for $\delta \lambda_{i}$ is equivalent to the differential equation

$\frac{d}{d t} \delta \lambda_{i}=-f_{x}\left(\chi_{i}\right) \delta \lambda_{i}-H_{x x}\left(\chi_{i}, \lambda_{i}\right) \delta x_{i}-H_{x u}\left(\chi_{i}, \lambda_{i}\right) \delta u_{i}$,

$\delta \lambda_{i}\left(t_{f}\right)=\left(g_{f}\right)_{x x}\left(x_{i}\left(t_{f}\right)\right) \delta x_{i}\left(t_{f}\right)$.

Proof: $\quad$ Due to $\lambda_{i}=\lambda\left[u_{i}\right]$, we have

$\frac{d}{d t} \lambda_{i}=-H_{x}\left(x_{i}, u_{i}, \lambda_{i}\right)^{\prime}$ and $\lambda_{i}\left(t_{f}\right)=\left(g_{f}\right)_{x}\left(x_{i}\left(t_{f}\right)\right)^{\prime}$.

Subtracting these equations respectively from (13) and (14), we have (16) and (17) respectively.

According to the SQQM, the solution estimate $\left(x_{i}, u_{i}, \lambda_{i}\right)$ is updated by $\left(x_{i+1}, u_{i+1}, \lambda_{i+1}\right)$ with $u_{i+1}=$ $u_{i}+\delta u_{i}, x_{i+1}=x\left[u_{i+1}\right]$ and $\lambda_{i+1}=\lambda\left[u_{i+1}\right]$. We make use of the notation $\zeta_{i}=\left[x_{i}^{\prime}, u_{i}^{\prime}, \lambda_{i}^{\prime}\right]^{\prime}$. Then, we have $\zeta_{i+1}=\left[x\left[u_{i+1}\right]^{\prime}, u_{i+1}^{\prime}, \lambda\left[u_{i+1}\right]^{\prime}\right]^{\prime}$. 


\section{Preliminaries for the convergence results}

Define $z(t)$ by $\left[x(t)^{\prime}, u(t)^{\prime}, \lambda(t)^{\prime}\right]^{\prime}$. Namely, $z(t) \in$ $\mathbf{R}^{2 n+m}$. We define the nonlinear operator $F$ by

$$
F(z)=\left[\begin{array}{l}
\frac{d}{d t} x-f(x, u) \\
\frac{d}{d t} \lambda+H_{x}(x, u, \lambda)^{\prime} \\
x\left(t_{0}\right)-x_{0} \\
\lambda\left(t_{f}\right)-\left(g_{f}\right)_{x}\left(x\left(t_{f}\right)\right) \\
H_{u}(x, u, \lambda)^{\prime}
\end{array}\right]
$$

Thus, the image $F(z)$ of $F$ is a composite of an $\mathbf{R}^{2 n+m}$ valued function and a vector in $\mathbf{R}^{2 n}$. Then, our original problem (4) is replaced by the problem of solving the equation

$$
F(z)=0 \text {. }
$$

It is also easy to observe that $z^{*}(t)=$ $\left[x^{*}(t)^{\prime}, u^{*}(t)^{\prime}, \lambda^{*}(t)^{\prime}\right]^{\prime}$ is a unique local solution of equation (19) (see (7)-(10)). We shall prove in this section that the iterative algorithm SQQM is asymptotically equivalent to the Newton's method

$$
z_{n+1}=z_{n}-\left\{\mathcal{D} F\left(z_{n}\right)\right\}^{-1} F\left(z_{n}\right)
$$

for solving (19) on the nonlinear operator $F$, where $\mathcal{D} F$ denotes the Fréchet derivative of $F$ (see Chapter 7 in [5] for its definition). It is easy to obtain the following preliminary results (see e.g., Chapters 7 and 9 in [5]).

From now on, we assume that the Newton's method defined by $(20)$ is well defined (i.e., $\mathcal{D} F\left(z_{i}\right)$ is invertible). In order to avoid an ambiguous expression in the following, we make use of the notation $\widetilde{\delta z_{i}}$ for $z_{i+1}-z_{i}$. Letting $x_{i}=x\left[u_{i}\right]$ and $\lambda_{i}=\lambda\left[u_{i}\right]$, we obtain the following result.

Proposition 3.1 The triplet $\left[\left(x_{i}+\delta x_{i}\right)^{\prime},\left(u_{i}+\right.\right.$ $\left.\left.\delta u_{i}\right)^{\prime},\left(\lambda_{i}+\delta \lambda_{i}\right)^{\prime}\right]^{\prime}$ whose increments were given by the $S Q Q M$ in Lemmas 2.1 and 2.3 determines the same function as $z_{i+1}$ obtained by Newton's method (20) for $z_{i}=\left[x\left[u_{i}\right]^{\prime}, u_{i}^{\prime}, \lambda\left[u_{i}\right]^{\prime}\right]^{\prime}$.

Proof: Equality (20) is equivalent to

$$
F\left(z_{i}\right)+\mathcal{D} F\left(z_{i}\right)\left(z_{i+1}-z_{i}\right)=F\left(z_{i}\right)+\mathcal{D} F\left(z_{i}\right) \widetilde{\delta z_{i}}=0
$$

Using the notation $\widetilde{\delta z_{i}}=\left[{\widetilde{\delta x_{i}}}^{\prime},{\widetilde{\delta u_{i}}}^{\prime},{\widetilde{\delta \lambda_{i}}}^{\prime}\right]^{\prime}$, the left hand side of (21) is equal to

$$
\left[\begin{array}{l}
\frac{d}{d t} x_{i}-f\left(\chi_{i}\right)+\frac{d}{d t} \widetilde{\delta x_{i}}-\frac{f_{x}}{\left(\chi_{i}\right) \widetilde{\delta x_{i}}-f_{u}\left(\chi_{i}\right) \widetilde{\delta u_{i}}} \\
\frac{d}{d t} \lambda_{i}+H_{x}\left(\chi_{i}, \lambda_{i}\right)^{\prime}+\frac{d}{d t} \widetilde{\delta \lambda_{i}}+H_{x x}\left(\chi_{i}, \lambda_{i}\right) \widetilde{\delta x_{i}} \\
\quad+H_{x u}\left(\chi_{i}, \lambda_{i}\right) \widetilde{\delta u_{i}}+f_{x}\left(\chi_{i}\right) \widetilde{\delta \lambda_{i}} \\
x_{i}\left(t_{0}\right)-x_{0}+\widetilde{\delta x_{i}}\left(t_{0}\right) \quad \widetilde{d_{i}}\left(t_{f}\right)-\left(g_{f}\right)_{x x}\left(x_{i}\left(t_{f}\right)\right) \widetilde{\delta x_{i}}\left(t_{f}\right) \\
\lambda_{i}\left(t_{f}\right)-\left(g_{f}\right)_{x}\left(x_{i}\left(t_{f}\right)\right)+\widetilde{\widetilde{\delta}_{i}} \\
H_{u}\left(\chi_{i}, \lambda_{i}\right)^{\prime}+H_{u x}\left(\chi_{i}, \lambda_{i}\right) \widetilde{\delta x_{i}}+H_{u u}\left(\chi_{i}, \lambda_{i}\right) \widetilde{\delta u_{i}}+f_{u}\left(\chi_{i}\right) \widetilde{\delta \lambda_{i}}
\end{array}\right]
$$

$$
=\left[\begin{array}{l}
\frac{d}{d t} \widetilde{\delta x_{i}}-f_{x}\left(\chi_{i}\right) \widetilde{\delta x_{i}}-f_{u}\left(\chi_{i}\right) \widetilde{\delta u_{i}} \\
\frac{d}{d t} \widetilde{\delta \lambda_{i}}+H_{x x}\left(\chi_{i}, \lambda_{i}\right) \widetilde{\delta x_{i}}+H_{x u}\left(\chi_{i}, \lambda_{i}\right) \widetilde{\delta u_{i}}+f_{x}\left(\chi_{i}\right) \widetilde{\delta \lambda_{i}} \\
\widetilde{\delta x_{i}}\left(t_{0}\right) \\
\widetilde{\gamma_{i+1}}\left(t_{f}\right)-\left(g_{f}\right)_{x x}\left(x_{i}\left(t_{f}\right)\right) \widetilde{\delta x_{i}}\left(t_{f}\right) \\
H_{u}\left(\chi_{i}, \widetilde{\gamma_{i+1}}\right)^{\prime}+H_{u x}\left(\chi_{i}, \lambda_{i}\right) \widetilde{\delta x_{i}}+H_{u u}\left(\chi_{i}, \lambda_{i}\right) \widetilde{\delta u_{i}}
\end{array}\right]
$$

where we used $x_{i}=x\left[u_{i}\right], \lambda_{i}=\lambda\left[u_{i}\right]$. Hence, due to Lemmas 2.1 and 2.3 , the existence of the solution $\left[\delta x_{i}^{\prime}, \delta u_{i}^{\prime}, \lambda_{i}^{\prime}\right]^{\prime}$ of (12)-(15) is equivalent to the existence of the solution $\widetilde{\delta z_{i}}=z_{i+1}-z_{i}$ of equation (21). Thus, the triplet $\left[\left(x_{i}+\delta x_{i}\right)^{\prime},\left(u_{i}+\delta u_{i}\right)^{\prime},\left(\lambda_{i}+\delta \lambda_{i}\right)^{\prime}\right]^{\prime}$ whose increments were given by the SQQM is equal to the function $z_{i+1}\left(=z_{i}+\widetilde{\delta z_{i}}\right)$ determined by the Newton's method (20) for $z_{i}=\left[x\left[u_{i}\right]^{\prime}, u_{i}{ }^{\prime}, \lambda\left[u_{i}\right]^{\prime}\right]^{\prime}$.

In the following, we show that the difference between the algorithm SQQM and the Newton's method is locally small enough so that the SQQM retains local convergence property similar to the Newton's method. From now on, we suppose that the assumption in Proposition 3.1 (i.e., $x_{i}=x\left[u_{i}\right]$ and $\lambda_{i}=\lambda\left[u_{i}\right]$ ) holds.

First, we give some definitions. For $\mathbf{R}^{d}$-valued continuous function $h(t)$, we define its norm $\|h\|$ by $\|h\|=\sum_{j=1}^{d} \sup _{t_{0} \leq t \leq t_{f}}\left|h^{(j)}(t)\right|$. It is well known that the space of $\mathbf{R}^{\bar{d}}$-valued continuous functions with this norm is a Banach space. We introduce the standard notation $q(x)=O(x)$ which means that $K=\limsup _{x \rightarrow 0}\|q(x)\| /\|x\|$ is finite for a function $q$ of variable $x$. The notation $q(x)=o(x)$ means that $K$, above, is zero. Similarly we define $O^{2}(\cdot)$ and $o^{2}(\cdot)$, with $\|q(x)\| /\|x\|^{2}$.

Recall that $\delta u_{i}, \delta x_{i}$ and $\delta \lambda_{i}$ are given in Lemmas 2.1 and 2.3. The functions $x_{i+1}$ and $\lambda_{i+1}$ are related to the SQQM and are defined respectively by $x\left[u_{i+1}\right]$ and $\lambda\left[u_{i+1}\right]$. In connection with the Newton's method, we make use of the notations with tilde. Thus, we use $\widetilde{x_{i+1}}:=x_{i}+\delta x_{i}$ and $\widetilde{\lambda_{i+1}}:=\lambda_{i}+\delta \lambda_{i}$. Here, due to Proposition 3.1, $\delta x_{i}$ and $\lambda_{i}$ are the same as those in Lemmas 2.1 and 2.3 under the above assumption. We also note that $z_{i+1}=z_{i}+\widetilde{\delta z_{i}}=\left[\widetilde{x_{i+1}}{ }^{\prime}, u_{i+1}{ }^{\prime}, \widetilde{\lambda_{i+1}}\right]^{\prime}$ for the Newton's method. We then obtain the next two lemmas in a standard way.

Lemma 3.2 We have the following estimates:

$$
\begin{aligned}
& \left\|\delta x_{i}\right\|=\left\|\widetilde{x_{i+1}}-x_{i}\right\|=O\left(\delta u_{i}\right) \\
& \left\|x_{i+1}-x_{i}\right\|=O\left(\delta u_{i}\right) .
\end{aligned}
$$

Proof: $\quad$ Since $\delta x_{i}$ satisfies the differential equation

$$
\frac{d}{d t} \delta x_{i}=f_{x}\left(\chi_{i}\right) \delta x_{i}+f_{u}\left(\chi_{i}\right) \delta u_{i}, \quad \delta x_{i}\left(t_{0}\right)=0,
$$

we obtain the first estimate by virtue of the GronwallBellman inequality.

$$
\begin{aligned}
& \text { Note that } \frac{d}{d t} x_{i}=f\left(\chi_{i}\right) \text { and } \\
& \begin{array}{l}
f\left(x_{i+1}, u_{i+1}\right)=f\left(x_{i}, u_{i}\right)+f_{x}\left(x_{i}, u_{i}\right)\left(x_{i+1}-x_{i}\right) \\
\quad+f_{u}\left(x_{i}, u_{i}\right)\left(u_{i+1}-u_{i}\right)+o\left(x_{i+1}-x_{i}\right)+o\left(\delta u_{i}\right) .
\end{array}
\end{aligned}
$$


Thus, using the notation $\xi=x_{i+1}-x_{i}$, we have

$$
\frac{d}{d t} \xi=f_{x}\left(\chi_{i}\right) \xi+f_{u}\left(\chi_{i}\right) \delta u_{i}+o(\xi)+o\left(\delta u_{i}\right) .
$$

Then, the second estimate immediately follows from the Gronwall-Bellman inequality.

Lemma 3.3 We have the following estimates:

$$
\begin{aligned}
& \left\|\widetilde{x_{i+1}}-x_{i+1}\right\|=O^{2}\left(\delta u_{i}\right), \\
& \left\|\widetilde{\lambda_{i+1}}-\lambda_{i+1}\right\|=O^{2}\left(\delta u_{i}\right) .
\end{aligned}
$$

Proof: $\quad$ Since

$$
\begin{aligned}
\frac{d}{d t} \widetilde{x_{i+1}} & =\frac{d}{d t} x_{i}+\frac{d}{d t} \delta x_{i} \\
& =f\left(x_{i}, u_{i}\right)+f_{x}\left(x_{i}, u_{i}\right) \delta x_{i}+f_{u}\left(x_{i}, u_{i}\right) \delta u_{i}
\end{aligned}
$$

we have, for each $j(1 \leq j \leq n)$,

$$
\begin{aligned}
& \frac{d}{d t} x_{i+1}^{(j)}=f^{(j)}\left(x_{i+1}, u_{i+1}\right) \\
&=f^{(j)}\left(\chi_{i}\right)+f^{(j)}{ }_{x}\left(\chi_{i}\right)\left(x_{i+1}-x_{i}\right)+f^{(j)}{ }_{u}\left(\chi_{i}\right) \delta u_{i} \\
& \quad+\frac{1}{2}\left(x_{i+1}-x_{i}\right)^{\prime} f^{(j)}{ }_{x x}\left(\chi_{i}\right)\left(x_{i+1}-x_{i}\right) \\
& \quad+\frac{1}{2}\left(x_{i+1}-x_{i}\right)^{\prime} f^{(j)}{ }_{x u}\left(\chi_{i}\right) \delta u_{i} \\
& \quad+\frac{1}{2} \delta u_{i}{ }^{\prime}{ }^{(j)}{ }_{u x}\left(\chi_{i}\right)\left(x_{i+1}-x_{i}\right)+\frac{1}{2} \delta u_{i}{ }^{\prime} f^{(j)}{ }_{u u}\left(\chi_{i}\right) \delta u_{i} \\
&=f^{(j)}\left(\chi_{i}\right)+f^{(j)}{ }_{x}\left(\chi_{i}\right)\left(\widetilde{x_{i+1}}-x_{i}\right)+f^{(j)}\left(\chi_{i}\right)\left(x_{i+1}-\widetilde{x_{i+1}}\right) \\
&+f^{(j)}{ }_{u}\left(\chi_{i}\right) \delta u_{i}+(\text { the same } 2 \text { nd order terms as above }) \cdot(27)
\end{aligned}
$$

Subtracting (27) from (26) and observing $\delta x_{i}=\widetilde{x_{i+1}}-$ $x_{i}$, we have the equality, for $\xi=\widetilde{x_{i+1}}-x_{i+1}$,

$$
\frac{d}{d t} \xi=f_{x}\left(x_{i}, u_{i}\right) \xi+O^{2}\left(\delta u_{i}\right)
$$

with $\xi\left(t_{0}\right)=0$. Here, we also used estimates (22) and (23) in Lemma 3.2. Thus, we obtain the first estimate (24) by virtue of the Gronwall-Bellman inequality.

For $\lambda_{i+1}$, we have

$$
\begin{aligned}
\frac{d}{d t} \lambda_{i+1}= & -H_{x}\left(x_{i+1}, u_{i+1}, \lambda_{i+1}\right)^{\prime} \\
= & -H_{x}\left(\chi_{i}, \lambda_{i}\right)^{\prime}-f_{x}\left(\chi_{i}\right)\left(\lambda_{i+1}-\lambda_{i}\right) \\
& -H_{x x}\left(\chi_{i}, \lambda_{i}\right)\left(x_{i+1}-x_{i}\right) \\
& -H_{x u}\left(\chi_{i}, \lambda_{i}\right) \delta u_{i}+O^{2}\left(\delta u_{i}\right) .
\end{aligned}
$$

We also note that

$\frac{d}{d t} \lambda_{i}=-H_{x}\left(\chi_{i}, \lambda_{i}\right)^{\prime}$

$\frac{d}{d t} \delta \lambda_{i}=-f_{x}\left(\chi_{i}\right) \delta \lambda_{i}-H_{x x}\left(\chi_{i}, \lambda_{i}\right) \delta x_{i}-H_{x u}\left(\chi_{i}, \lambda_{i}\right) \delta u_{i}(30$

hold. Computing (29) plus (30) minus (28), we have

$$
\begin{aligned}
\frac{d}{d t}\left(\widetilde{\lambda_{i+1}}-\lambda_{i+1}\right)= & -f_{x}\left(\chi_{i}\right)\left(\widetilde{\lambda_{i+1}}-\lambda_{i+1}\right) \\
& -H_{x x}\left(\chi_{i}, \lambda_{i}\right)\left(\widetilde{x_{i+1}}-x_{i+1}\right)+O^{2}\left(\delta u_{i}\right) \\
= & -f_{x}\left(\chi_{i}\right)\left(\widetilde{\lambda_{i+1}}-\lambda_{i+1}\right)+O^{2}\left(\delta u_{i}\right)
\end{aligned}
$$

by virtue of $(22),(23)$ and (24). For the terminal condition, we have

$$
\begin{aligned}
& \lambda_{i+1}\left(t_{f}\right)=\left(g_{f}\right)_{x}\left(x_{i+1}\left(t_{f}\right)\right)^{\prime}, \\
& \lambda_{i}\left(t_{f}\right)=\left(g_{f}\right)_{x}\left(x_{i}\left(t_{f}\right)\right)^{\prime}, \\
& \delta \lambda_{i}\left(t_{f}\right)=\left(g_{f}\right)_{x x}\left(x_{i}\left(t_{f}\right)\right) \delta x_{i}\left(t_{f}\right) .
\end{aligned}
$$

Computing (33) plus (34) minus (32), we have

$$
\widetilde{\lambda_{i+1}}\left(t_{f}\right)-\lambda_{i+1}\left(t_{f}\right)=O\left(\widetilde{x_{i+1}}-x_{i+1}\right)=O^{2}\left(\delta u_{i}\right) .
$$

Then, due to (31) and (35), the second estimate (24) immediately follows from the Gronwall-Bellman inequality.

We now recall that, for $z_{i}=\left[x_{i}{ }^{\prime}, u_{i}{ }^{\prime}, \lambda_{i}{ }^{\prime}\right]^{\prime}, z_{i+1}=$ $\left[\left(x_{i}+\delta x_{i}\right)^{\prime},\left(u_{i}+\delta u_{i}\right)^{\prime}, \quad\left(\lambda_{i}+\delta \lambda_{i}\right)^{\prime}\right]^{\prime}$ is related to the Newton's method, and $\zeta_{i+1}=\left[x_{i+1}{ }^{\prime}, u_{i+1}{ }^{\prime}, \lambda_{i+1}{ }^{\prime}\right]^{\prime}$ is to the algorithm SQQM. When $x_{i}=x\left[u_{i}\right]$ and $\lambda_{i}=\lambda\left[u_{i}\right]$ hold, we obtain the next proposition.

Proposition 3.4 When $\zeta_{i}=z_{i}=\left[x\left[u_{i}\right]^{\prime}, u_{i}{ }^{\prime}, \lambda\left[u_{i}\right]^{\prime}\right]^{\prime}$, the algorithm $S Q Q M$ has the form

$$
\zeta_{i+1}=\zeta_{i}-\left\{\mathcal{D} F\left(\zeta_{i}\right)\right\}^{-1} F\left(\zeta_{i}\right)+a_{i}\left(\zeta_{i}\right),
$$

where $a_{i}\left(\zeta_{i}\right)$ satisfies the following inequalities: there exist $\rho_{0}>0$ and $R>0$ such that, for any $i \geq 0$,

$$
\left\|a_{i}\left(\zeta_{i}\right)\right\| \leq R\left\|\delta u_{i}\right\|^{2} \quad \text { if }\left\|\delta u_{i}\right\| \leq \rho_{0},
$$

and

$$
\begin{gathered}
\left\|a_{i}\left(\zeta_{i}\right)\right\| \leq R \min \left\{\left\|\zeta_{i+1}-\zeta_{i}\right\|^{2},\left\|z_{i+1}-z_{i}\right\|^{2}\right\} \\
\text { if }\left\|\zeta_{i+1}-\zeta_{i}\right\| \leq \rho_{0} \text { or }\left\|z_{i+1}-z_{i}\right\| \leq \rho_{0} .
\end{gathered}
$$

Moreover, we also have that, for any $\tau>0$, there exists $\rho_{1}=\rho_{1}(\tau)>0$ such that, for any $i \geq 0$, the inequality

$$
\left\|a_{i}\left(\zeta_{i}\right)\right\| \leq \tau \alpha\left\|\zeta_{i+1}-\zeta_{i}\right\|
$$

holds if $\left\|\zeta_{i+1}-\zeta_{i}\right\| \leq \rho_{1}$ or $\left\|z_{i+1}-z_{i}\right\| \leq \rho_{1}$.

Proof: $\quad$ Estimates (24) and (25) in Lemma 3.3 implies

$$
\zeta_{i+1}=z_{i+1}+O^{2}\left(\delta u_{i}\right)
$$

On the other hand, in view of Lemma 2.3, (22) in Lemma 3.2 and the Gronwall-Bellman inequality, we have

$$
\left\|z_{i+1}-z_{i}\right\|=O\left(\delta u_{i}\right) \text { and }\left\|\zeta_{i+1}-\zeta_{i}\right\|=O\left(\delta u_{i}\right) .
$$

Due to (40) and (41), we have (37) and (38). Moreover, (39) immediately follows from (38).

\section{Local convergence of the SQQM}

In this section, we discuss local convergence of the SQQM. First, we prove linear convergence in Theorem 4.2 and then prove quadratic convergence in Theorem 4.3 under stronger assumptions. We distinguish the two sequences $\left\{\zeta_{n}\right\}$ and $\left\{z_{n}\right\}$ as before: $\left\{\zeta_{n}\right\}$ corresponds to the sequence generated by the SQQM and $\left\{z_{n}\right\}$ corresponds to the Newton's method. We explicitly state the following commonly used assumptions. 
Assumption $4 \quad F$ is continuously Fréchet differentiable on a convex neighborhood $D_{0}$ of $z^{*}$. Thus, $F$ satisfies the following Lipschitz continuity:

$$
\|F(z)-F(\bar{z})\| \leq K_{0}\|z-\bar{z}\| \quad \text { for } z, \bar{z} \in D_{0},
$$

for some constant $K_{0}$. Moreover, the Fréchet derivative $\mathcal{D} F(z)$ is invertible and satisfies the inequality

$$
\left\|\{\mathcal{D} F(z)\}^{-1}\right\| \leq M_{0} \quad \text { for } z \in D_{0} .
$$

Introducing the notation

$$
T(z)=z-\{\mathcal{D} F(z)\}^{-1} F(z),
$$

we can write iterative process (36) as

$$
\zeta_{n+1}=T\left(\zeta_{n}\right)+a_{i}\left(\zeta_{n}\right)
$$

In order to prove local convergence of (42), we need the following standard assumption.

Assumption 5 There is a convex closed neighborhood $D_{1}\left(\subset D_{0}\right)$ of $z^{*}$ such that $F$ is twice continuously Fréchet differentiable on $D_{1}$ and the following Lipschitz continuty

$$
\left\|\{\mathcal{D} F(z)\}^{-1}-\{\mathcal{D} F(\bar{z})\}^{-1}\right\| \leq M_{1}\|z-\bar{z}\|,
$$

holds for $z, \bar{z} \in D_{1}$ and a constant $M_{1}>0$.

Under Assumption 5, we obtain the following lemma due to a simple adaptation of Theorem 3.3.1 in [12] (which was proved on $\mathbf{R}^{n}$ ).

\section{Lemma 4.1 (Contraction mapping property)}

For a convex closed neighborhood $D_{2}\left(\subset D_{1}\right)$ of $z^{*}$, the operator $T$ is a contraction mapping on $D_{2}$ under Assumption 5.

Making use of Lemma 4.1, we obtain the following local convergence of iterative process (42) at a linear rate.

Theorem 4.2 (Linear convergence) The sequence $\left\{\zeta_{n}\right\}$ generated by the SQQM converges locally to $z^{*}$ at a linear rate under the prescribed assumptions and the conditions in Lemma 4.1.

Proof: $\quad$ Notice that we have

$$
\zeta_{n+1}=T\left(\zeta_{n}\right)+a_{i}\left(\zeta_{n}\right)
$$

where $T$ and $a_{n}$ respectively satisfy

$$
\begin{aligned}
& T\left(z^{*}\right)=z^{*} \quad \text { (i.e., } z^{*} \text { is a fixed point), } \\
& \|T(z)-T(\bar{z})\| \leq \alpha\|z-\bar{z}\| \quad\left(z, \bar{z} \in D_{2}\right),
\end{aligned}
$$

for some positive constant $\alpha<1$ (due to Lemma 4.1) and $a_{n}\left(z^{*}\right)=0$. Here, the fixed point property of $z^{*}$ with respect to $T$ follows from the property $F\left(z^{*}\right)=0$. The last equality follows from the fact that $\zeta_{n+1}=$ $z_{n+1}=z^{*}$ holds if $\zeta_{n}=z^{*}$ (see Remark 2.2). We assume $\rho_{0}>0$ small enough to satisfy $R \rho_{0} \leq 1$ by redefining $\rho_{0}>0$ with a smaller value if necessary. Choose $\tau>0$ small enough to assure the inequality

$$
0<\alpha \frac{1+\tau}{1-\tau \alpha}<1
$$

Let $\xi>0$ be small enough to ensure that $\left\{z:\left\|z-z^{*}\right\|<\right.$ $\xi\} \subset D_{2}$. We choose $\rho_{2}\left(\leq \min \left\{\rho_{0}, \rho_{1}\right\}\right)$ so that

$$
\frac{\rho_{2}}{2 K_{0} M_{0}}<\xi
$$

Let $\zeta_{0}$ be an initial point in $D_{2}$ with $\left\|\zeta_{0}-z^{*}\right\| \leq$ $\rho_{2} /\left(2 K_{0} M_{0}\right)$. Since the inequality

$$
\left\|F\left(\zeta_{0}\right)\right\|=\left\|F\left(\zeta_{0}\right)-F\left(z^{*}\right)\right\| \leq K_{0}\left\|\zeta_{0}-z^{*}\right\| \leq \rho_{2} /\left(2 M_{0}\right)
$$

holds, we have

$\left\|z_{1}-z_{0}\right\|=\left\|\left\{\mathcal{D} F\left(\zeta_{0}\right)\right\}^{-1} F\left(\zeta_{0}\right)\right\| \leq M_{0}\left\|F\left(\zeta_{0}\right)\right\| \leq \rho_{2} / 2$,

and so

$$
\left\|a_{0}\left(\zeta_{0}\right)\right\| \leq R \rho_{0}\left\|z_{1}-z_{0}\right\| \leq\left\|z_{1}-z_{0}\right\| \leq \rho_{2} / 2,
$$

by virtue of (38). Thus, we have

$$
\left\|\zeta_{1}-\zeta_{0}\right\| \leq M_{0}\left\|F\left(\zeta_{0}\right)\right\|+\left\|a_{0}\left(\zeta_{0}\right)\right\| \leq \rho_{2},
$$

using (48) and (49). Hence, we have

$$
\left\|a_{0}\left(\zeta_{0}\right)\right\| \leq \tau \alpha\left\|\zeta_{1}-\zeta_{0}\right\|
$$

Due to (43) and (44), we have

$$
\begin{aligned}
\left\|\zeta_{1}-z^{*}\right\| & =\left\|T\left(\zeta_{0}\right)-T\left(z^{*}\right)+a_{0}\left(\zeta_{0}\right)\right\| \\
& \leq\left\|T\left(\zeta_{0}\right)-T\left(z^{*}\right)\right\|+\left\|a_{0}\left(\zeta_{0}\right)\right\| \\
& \leq \alpha\left\|\zeta_{0}-z^{*}\right\|+\tau \alpha\left\|\zeta_{1}-\zeta_{0}\right\| \\
& \leq \alpha\left\|\zeta_{0}-z^{*}\right\|+\tau \alpha\left(\left\|\zeta_{1}-z^{*}\right\|+\left\|\zeta_{0}-z^{*}\right\|\right),
\end{aligned}
$$

where Lemma 4.1 and inequality (50) are applied to the second inequality. Hence, we have

$$
\left\|\zeta_{1}-z^{*}\right\| \leq \bar{\alpha}\left\|\zeta_{0}-z^{*}\right\|
$$

with $\bar{\alpha}:=\alpha(1+\tau) /(1-\tau \alpha) \in(0,1)$ (due to $(46))$. Hence, $\zeta_{1}$ satisfies $\left\|\zeta_{1}-z^{*}\right\| \leq \rho_{2} /\left(2 K_{0} M_{0}\right)$ and also $\zeta_{1} \in D_{2}$ (see (47)). Thus, $\zeta_{1}$ satisfies the requirement which was imposed on $\zeta_{0}$ in the first part of this proof.

For a general natural number $n$, we assume that $\zeta_{n}$ be a point in $D_{2}$ with $\left\|\zeta_{n}-z^{*}\right\| \leq \rho_{2} /\left(2 K_{0} M_{0}\right)$. Then, by exactly the same proof as above, we can prove

$$
\left\|\zeta_{n+1}-z^{*}\right\| \leq \bar{\alpha}\left\|\zeta_{n}-z^{*}\right\|
$$

and also $\left\|\zeta_{n+1}-z^{*}\right\| \leq \rho_{2} /\left(2 K_{0} M_{0}\right)$. Namely, we proved the conclusion by induction.

We prove quadratic convergence of the SQQM under stronger assumptions which are similar to those used by Kantorovich (see Theorem 5 in Chapter 18 of [4]). 
Theorem 4.3 (Quadratic convergence) Let the sequence $\left\{\zeta_{n}\right\}$ be generated by the SQQM. Suppose the following conditions are satisfied besides all the assumptions already stated:

$$
\left\|F\left(\zeta_{0}\right)\right\| \leq \eta \quad \text { and } \quad\left\|\mathcal{D}^{2} F(z)\right\| \leq L \quad\left(z \in D_{0}\right) .
$$

We define $B(>0)$ by $B:=(1-\tau \alpha)^{-1} M_{0}$, where $\alpha$ and $\tau$ were defined in the Proof of Theorem 4.2. Suppose also that

$$
\begin{aligned}
& h:=\left(L+2 M_{2} R\right) B^{2} \eta<2, \\
& r:=B \eta \sum_{k=0}^{\infty}(h / 2)^{2^{k}-1}<\xi, \quad B \eta \leq \rho_{0},
\end{aligned}
$$

where $\xi>0$ was defined in the Proof of Theorem 4.2, $\rho_{0}$ was given in Proposition 3.4, and $M_{2}$ is a positive constant such that the inequality $\mid \mathcal{D} F(z) \| \leq M_{2}(z \in$ $D_{0}$ ) holds (owing to (51)).

Then, the sequence $\left\{\zeta_{n}\right\}$ with $\zeta_{0} \in D_{2}$ converges to $z^{*}$. Moreover, the rate of convergence is given by

$$
\left\|\zeta_{n}-z^{*}\right\| \leq B \eta(h / 2)^{2^{n}-1} /\left\{1-(h / 2)^{2^{n}}\right\} .
$$

Proof: $\quad$ Let $\zeta_{0}$ be an initial point in $D_{2}$. Then, we have

$$
\left\|z_{1}-z_{0}\right\|=\left\|\left\{\mathcal{D} F\left(\zeta_{0}\right)\right\}^{-1} F\left(\zeta_{0}\right)\right\| \leq M_{0} \eta<B \eta \leq \rho_{0} .
$$

Thus, making use of upper bound (38), we obtain

$$
\begin{aligned}
& \left\|\zeta_{1}-\zeta_{0}\right\|=\left\|-\left\{\mathcal{D} F\left(\zeta_{0}\right)\right\}^{-1} F\left(\zeta_{0}\right)+a_{0}\left(\zeta_{0}\right)\right\| \\
& \quad \leq M_{0}\left\|F\left(\zeta_{0}\right)\right\|+\left\|a_{0}\left(\zeta_{0}\right)\right\| \leq M_{0} \eta+\tau \alpha\left\|\zeta_{1}-\zeta_{0}\right\|,
\end{aligned}
$$

and so $\left\|\zeta_{1}-\zeta_{0}\right\| \leq B \eta$.

We note the equality

$$
\begin{aligned}
\Gamma_{0}\left(F\left(\zeta_{1}\right)\right)= & \Gamma_{0}\left(F\left(\zeta_{0}\right)\right)+\Gamma_{0} \mathcal{D} F\left(\zeta_{0}\right)\left(\zeta_{1}-\zeta_{0}\right) \\
& +\int_{\zeta_{0}}^{\zeta_{1}} \Gamma_{0} \mathcal{D}^{2} F(\zeta)\left(\zeta_{1}-\zeta, \cdot\right) d \zeta \\
= & \int_{\zeta_{0}}^{\zeta_{1}} \Gamma_{0} \mathcal{D}^{2} F(\zeta)\left(\zeta_{1}-\zeta, \cdot\right) d \zeta+a_{0}\left(\zeta_{0}\right)
\end{aligned}
$$

holds with $\Gamma_{0}=\left\{\mathcal{D} F\left(\zeta_{0}\right)\right\}^{-1}$. So we have

$$
F\left(\zeta_{1}\right)=\int_{\zeta_{0}}^{\zeta_{1}} \mathcal{D}^{2} F(\zeta)\left(\zeta_{1}-\zeta, \cdot\right) d \zeta+\mathcal{D} F\left(\zeta_{0}\right) a_{0}\left(\zeta_{0}\right)
$$

and hence

$$
\begin{aligned}
\left\|F\left(\zeta_{1}\right)\right\| & \leq L \int_{0}^{B \eta}(B \eta-t) d t+\left\|\mathcal{D} F\left(\zeta_{0}\right)\right\|\left\|a_{0}\left(\zeta_{0}\right)\right\| \\
& \leq \frac{1}{2} L B^{2} \eta^{2}+M_{2} R\left\|\zeta_{1}-\zeta_{0}\right\|^{2} \\
& \leq \frac{1}{2} L B^{2} \eta^{2}+M_{2} R B^{2} \eta^{2}=\frac{\left(L+2 M_{2} R\right) B^{2} \eta}{2} \eta .
\end{aligned}
$$

Thus, using the notations $h=\left(L+2 M_{2} R\right) B^{2} \eta$ and $\eta_{1}:=\frac{h \eta}{2}$, we have $\left\|F\left(\zeta_{1}\right)\right\| \leq \eta_{1}$.
We note that the inequality

$$
\left\|\widetilde{z_{2}}-\zeta_{1}\right\|=\left\|\left\{\mathcal{D} F\left(\zeta_{1}\right)\right\}^{-1} F\left(\zeta_{1}\right)\right\| \leq M_{0} \eta_{1}<B \eta \leq \rho_{0}
$$

holds since $h<2$ implies $\eta_{1}<\eta$, where $\widetilde{z_{2}}$ is the function determined by the Newton's method from the function $\zeta_{1}$.

With the notation $h_{1}:=\left(L+2 M_{2} R\right) B^{2} \eta_{1}$, we have

$$
h_{1}=\frac{\left(L+2 M_{2} R\right) B^{2} \eta}{2} h=\frac{h^{2}}{2} .
$$

Hence, due to (38) and (53), we obtain

$$
\begin{aligned}
\left\|\zeta_{2}-\zeta_{1}\right\| & =\left\|-\left\{\mathcal{D} F\left(\zeta_{1}\right)\right\}^{-1} F\left(\zeta_{1}\right)+a_{1}\left(\zeta_{1}\right)\right\| \\
& \leq M_{0}\left\|F\left(\zeta_{1}\right)\right\|+\left\|a_{1}\left(\zeta_{1}\right)\right\| \\
& \leq M_{0} \eta_{1}+\tau \alpha\left\|\zeta_{2}-\zeta_{1}\right\|
\end{aligned}
$$

and hence $\left\|\zeta_{2}-\zeta_{1}\right\| \leq B \eta_{1}$.

Using the notations

$$
h_{k}:=\left(L+2 M_{2} R\right) B^{2} \eta_{k} \text { and } \eta_{k+1}:=\frac{h_{k} \eta_{k}}{2},
$$

and assuming the inequality

$$
\left\|\zeta_{k+1}-\zeta_{k}\right\| \leq B \eta_{k}
$$

we can prove the inequalities

$$
\left\|F\left(\zeta_{k+1}\right)\right\| \leq \eta_{k+1} \text { and }\left\|\zeta_{k+2}-\zeta_{k+1}\right\| \leq B \eta_{k+1} .
$$

From the above definitions of $h_{k}$ and $\eta_{k}$, we obtain

$$
\begin{aligned}
h_{k} & =\left(L+2 M_{2} R\right) B^{2} \eta_{k-1} h_{k-1} / 2 \\
& =\frac{h_{k-1}^{2}}{2}=2\left(\frac{h_{k-1}}{2}\right)=\cdots=2\left(\frac{h}{2}\right)^{2^{k}}
\end{aligned}
$$

for $k=0,1, \cdots, n$, and so

$$
\begin{aligned}
\eta_{n} & =\frac{h_{n-1} \eta_{n-1}}{2}=\frac{h_{n-1} h_{n-2} \eta_{n-2}}{4}=\cdots \\
& =\frac{h_{n-1} h_{n-2} \cdots h_{0}}{2^{n}} \eta=\left(\frac{h}{2}\right)^{2^{n}-1} \eta .
\end{aligned}
$$

Hence, the inequality

$$
\left\|\zeta_{n+1}-\zeta_{n}\right\| \leq B \eta\left(\frac{h}{2}\right)^{2^{n}-1}
$$

holds. The remainder of the proof is exactly same as the Proof of Theorem 5 in Chapter 18 of [4]. Hence, it is omitted.

\section{ACKNOWLEDGMENTS}

The first author thanks Drs. I. N. Katz and H. Mukai of Washington University for their generous support while he was there. He also would like to thank Prof. M. Hitsuda of Kumamoto University for kindly providing some references. 


\section{References}

[1] T. Basar and G. J. Olsder, Dynamic Noncooperative Game Theory, second edition, Academic Press, New York, New York, 1995.

[2] A. E. Bryson, Jr. and Y. C. Ho, Applied Optimal Control, Blaisdell Publishing Company, Waltham, Massachusetts, 1969.

[3] F. Caliskan et al., "Game estimators for air combat games with unknown enemy inputs", in Proc. American Control Conf., pp. 5381-5387, 2003.

[4] L. V. Kantorovich and G. P. Akilov, Functional Analysis, Second Edition, Pergamon Press, Oxford, England, 1982.

[5] D. G. Luenberger, Optimization by Vector Space Method, John Wiley and Sons, New York, New York, 1969.

[6] H. Mukai et al., "Sequential linear quadratic method for differential games", in Proc. 2nd DARPA-JFACC Symposium on Advances in Enterprise Control, pp. 159-168, Minneapolis, MN, July 2000.

[7] H. Mukai et al., "Game-theoretic linear-quadratic method for air mission control", in Proc. 39th IEEE Conf. Decision and Control, pp. 2574-2580, Sydney, Australia, Dec, 2000.

[8] H. Mukai et al., "Sequential quadratic methods for differential games", in Proc. American Control Conf., pp. 182-189, Washington, D.C., June 2001.

[9] S. M. Robinson, A quadratically-convergent algorithm for general nonlinear programming problems, Math. Programming 3, 145-156 (1972).

[10] S. M. Robinson, Perturbed Kuhn-Tucker points and rates of convergence for a class of nonlinearprogramming algorithms, Math. Programming 7, 1-16 (1974).

[11] I. Tunay, J. Goodwin, and H. Mukai, "Markov Chain Combat Models for Prediction of Attrition and Mission Control", Proc. of the International Military Conf. of the INFORMS Military Application Society, 15 pages, San Antonio, Texas, November 2000.

[12] Y. Shinohara, Foundations of Numerical Analysis,(in Japanese), Nisshin Shuppan, Tokyo, Japan, 1978.

[13] R. B. Wilson, A simplicial algorithm for concave programming, Ph.D. Dissertation, Graduate School of Bussiness Administration, Harvard University, Cambridge, Mass., 1963. 